\title{
Theory of time-dependent propagation in multimode lightguides*
}

\author{
Bruno Crosignani ${ }^{\dagger}$ and Charles H. Papas \\ California Institute of Technology, Pasadena, California 91125 \\ Paolo Di Porto \\ Fondazione Ugo Bordoni, Istituto Superiore Poste e Telecomunicazioni, Viale Europa, Roma, Italy \\ (Received 6 December 1976; revision received 22 July 1977)
}

\begin{abstract}
A coupled system of equations governing the propagation of a signal in a statistical ensemble of multimode optical fibers is presented. It describes, besides the usual average modal powers, the evolution of the interference terms between the mode amplitudes and of the modal power fluctuations. Our procedure allows us to treat the general nonstationary nonmonochromatic case of an arbitrary signal fed into the lightguide by a source possessing a finite spectral bandwidth. The introduction of modal power fluctuations permits us to establish a theorem connecting the value of the modal power, averaged over the fiber ensemble, with the actual one concerning a single fiber. These two values coincide, in the polychromatic case, for large values of the fiber length, thus providing the main result of the paper, that is the justification of the statistical approach to the problem of propagation. Furthermore, the analysis of the interference terms presents evidence for the difference between the propagation of an amplitude-modulated and a frequency-modulated signal.
\end{abstract}

\section{INTRODUCTION}

Multimode optical fibers with large-diameter core are particularly useful in connection with sources possessing intermediate degrees of spatial coherence, such as lightemitting diodes and laser diodes. These sources have a finite bandwidth $\delta \nu$, and, accordingly, the problem of propagation in the fiber cannot be simplified by assuming a monochromatic input. Indeed, a theory which takes into account the lack of monochromaticity (or the finite coherence time $t_{c}=1 / \delta \nu$ ) is necessary.

The description of the propagation of an electromagnetic signal in a multimode lightguide in the presence of mode coupling is a statistical one. This is a consequence of the fact that the guiding structure unavoidably presents some imperfections responsible for the coupling among the modes, whose randomness, together with the analytical complexity of the exact deterministic problem, leads in a natural way to the statistical approach. It consists of an introduction of an ensemble of macroscopically similar fibers, which differ among themselves only for the random behavior of the microscopic imperfections, and we consider the values of all the significant quantities averaged over this ensemble. ${ }^{1}$ In this frame, the main emphasis has been placed on the evaluation of the average power carried by each propagating mode, both in the case of a monochromatic ${ }^{1}$ and of a polychromatic signal. ${ }^{2,3}$

However, it is hardly necessary to remark, as always when dealing with a statistical approach, about the relevance of the fluctuations over the average value, since only their (relative) smallness assures that the average value of a given quantity can be safely assumed as the actual one. The fluctuations have been evaluated, only in the monochromatic case, ${ }^{1,4}$ with a rather disap-pointing result. More precisly, it has been found that the normalized mean-square value of the power carried by each mode approaches 2 for long-traveled distances, so that there is a $100 \%$ uncertainty on the actual value of this quantity in a single fiber once the average value is known.

The above discussion gives evidence to the necessity of refining the theory of propagation in a multimode optical fiber in two ways. First, the existing treatment only deals with the average power per mode, which is connected with the square value of the corresponding mode amplitude, while a more complete description also requires the study of the evolution of the correlation terms between different mode amplitudes. In the course of the paper it will be shown that these are the only terms influenced by a frequency modulation of the input signal, an operation which, conversely, does not affect the behavior of the power per mode. Second, one needs a more complete investigation of the fluctuations, capable of covering the nonmonochromatic case. In fact, it will be proved that the finite bandwidth of the source has the positive consequence of reducing the magnitude of the fluctuations so that, for long-traveled distances, the average power per mode does coincide with the actual value in a single fiber.

The mentioned results are obtained in the frame of a general theory of nonstationary propagation, dealing with a carrier possessing a finite bandwidth and modulated in an arbitrary way, in which the complete description of the propagation and of the associated fluctuations is obtained in the form of a closed system of differential equations connecting all the correlation terms between mode amplitudes up to the fourth order. The present treatment generalizes that of Marcuse ${ }^{1}$ since it is applicable to the propagation of a polychromatic signal, a case which substantially differs from that relative to the monochromatic signal, as it is for example shown by the drastic change in the behavior of the fluctuations, which improves the predictability of the actual value of the single-mode power in the case of finite bandwidth.

\section{PROPAGATION IN A SINGLE FIBER}

The transverse part of a monochromatic electromagnetic field of angular frequency $\omega$, propagating in the $z$ direction along the axis of a (nearly) cylindrical dielectric fiber, can be expressed ${ }^{1}$

$$
\begin{aligned}
\mathbf{E}(\mathrm{r}, z, t)= & \sum_{m} \mathrm{E}_{m}(\mathrm{r}, \omega) c_{m}(z, \omega) \\
& \times \exp \left[i \omega t-i \beta_{m}(\omega) z\right],
\end{aligned}
$$


where $\mathbf{E}_{m}(\mathbf{r}, \omega)$ is the transverse electric field vector of the $m$ th mode of the ideal cylindrical guiding structure, $\beta_{m}(\omega)$ is its relative propagation constant, and the $c_{m}$ 's are suitable expansion coefficients. In writing Eq. (1), we have assumed that the electric field can be expressed in terms of the forward-traveling guided modes alone; and we have allowed the expansion coefficients $c_{m}$ to be $z$ dependent in order to take into account the departure of the fiber shape from the ideal one to which the mode configurations $\mathbf{E}_{m}$ pertain. Here $\mathbf{r}=(x, y)$ indicates the coordinates in a plane transverse to $z$ and one needs to consider only the transverse part of the electric field $\mathrm{E}(\mathrm{r}, z, t)$ and of the magnetic field

$$
\begin{aligned}
\mathrm{H}(\mathrm{r}, z, t)= & \sum_{m} \mathrm{H}_{m}(r, \omega) c_{m}(z, \omega) \\
& \times \exp \left[i \omega t-i \beta_{m}(\omega) z\right]
\end{aligned}
$$

for the purpose of evaluating the power carried by the field.

Equations (1) and (2) can be easily generalized to the nonmonochromatic case. In particular, if, as it is usually the case, the bandwidth $\delta \omega$ of the field fulfills the relation

$$
\delta \omega / \omega_{0} \ll 1,
$$

where $\omega_{0}$ is the central frequency, one can write

$$
\begin{aligned}
\mathbf{E}(\mathbf{r}, z, t)= & \sum_{m} \mathrm{E}_{m}\left(\mathbf{r}, \omega_{0}\right) \int d \omega c_{m}(z, \omega) \\
& \times \exp \left[i \omega t-i \beta_{m}(\omega) z\right]
\end{aligned}
$$

and an analogous expression for $H$. The complex Poynting vector is given by ${ }^{5}$

$$
\mathrm{S}=\frac{1}{2}\left\langle\mathbf{E} \times \mathrm{H}^{*}\right\rangle_{\mathrm{av}},
$$

where \langle\rangle$_{\mathrm{a}}$ indicates the averaging operation over the fluctuations of the source. It is worth noting that the electromagnetic field possesses, in general, fast fluctuations associated with the carrier (that is with the source) and a slow variation associated with the superimposed signal. The averaging operation indicated as \langle\rangle$_{\text {av }}$ refers to the statistical ensemble pertinent to the source. Whenever the source is stationary, and the ergodic hypothesis is justified, ${ }^{6}$ the quantity $P_{t}$ in Eq. (6) represents the power averaged over a time of the order of $2 \pi /$ $\delta \omega$ and still retains the slow-time variation.

The power carried by the field through an arbitrary fiber section $z=$ const can be expressed

$$
P_{t}=\operatorname{Re} \int_{-\infty}^{+\infty} d x \int_{-\infty}^{+\infty} d y \mathbf{s} \cdot \mathrm{e}_{z}
$$

where $\mathrm{e}_{z}$ is the unit vector in the $z$ direction and " $\mathrm{Re}$ " means "real part of." Taking advantage of the orthogonality relations between the guided modes ${ }^{1}$

$$
\int_{-\infty}^{+\infty} d x \int_{-\infty}^{+\infty} d y \mathrm{e}_{z} \cdot \mathrm{E}_{n}\left(r, \omega_{0}\right) \times \mathrm{H}_{m}^{*}\left(\mathrm{r}, \omega_{0}\right)=2 P \delta_{m n},
$$

where $P$ is a positive normalization constant and $\delta_{m n}$ is Kronecker's symbol, one obtains through Eqs. (4)-(7),

$P_{t}=P \sum_{m} \int d \omega \int d \omega^{\prime}\left\langle c_{m}(z, \omega) c_{m}^{*}\left(z, \omega^{\prime}\right)\right\rangle_{\mathrm{av}}$

$$
\times \exp \left\{i\left(\omega-\omega^{\prime}\right) t-i\left[\beta_{m}(\omega)-\beta_{m}\left(\omega^{\prime}\right)\right] z\right\} \equiv \sum_{m} P_{m}(z, t)
$$

where $P_{m}(z, t)$ is a positive quantity, as it is immediately verified by inspection, which can be interpreted as the electromagnetic power carried by the $m$ th mode.

If mode coupling is absent, one has

$$
\begin{aligned}
c_{m}(z, \omega)= & c_{m}(0, \omega)=\frac{1}{4 \pi P} \int_{-\infty}^{+\infty} d t e^{-i \omega t} \\
& \times \int_{-\infty}^{+\infty} d x \int_{-\infty}^{+\infty} d y \mathrm{e}_{z} \cdot \mathrm{E}(\mathrm{r}, 0, t) \times \mathrm{H}_{m}^{*}\left(\mathrm{r}, \omega_{0}\right) \\
= & \frac{1}{4 \pi P} \int_{-\infty}^{+\infty} d t e^{-i \omega t} e^{i \omega_{0} t} F(t) \\
& \times \int_{-\infty}^{+\infty} d x \int_{-\infty}^{+\infty} d y \mathrm{e}_{z} \cdot \mathrm{G}(\mathrm{r}) \times \mathrm{H}_{m}^{*}\left(\mathrm{r}, \omega_{0}\right)
\end{aligned}
$$

having assumed, for the sake of simplicity, the electric field at $z=0$ to be of the form

$$
E(\mathrm{r}, 0, t)=e^{i \omega_{0} t} F(t) \mathrm{G}(\mathrm{r}) \text {. }
$$

Taking advantage of Eq. (3), one can approximately write

$$
\beta_{m}(\omega)=\beta_{m}\left(\omega_{0}\right)+\left(\omega-\omega_{0}\right) / V_{m},
$$

with

$$
V_{m}^{-1}=\left[d \beta_{m}(\omega) / d \omega\right]_{\omega=\omega_{0}}
$$

and Eq. (8) yields

$$
\begin{aligned}
P_{t}= & \frac{1}{4 P} \sum_{m}\left\langle\left|F\left(t-\frac{z}{V_{m}}\right)\right|^{2}\right\rangle_{\mathrm{av}} \\
& \times\left|\int_{-\infty}^{+\infty} d x \int_{-\infty}^{+\infty} d y \mathrm{G}(\mathrm{r}) \times \mathrm{H}_{m}^{*}\left(\mathrm{r}, \omega_{0}\right) \cdot \mathrm{e}_{z}\right|^{2} .
\end{aligned}
$$

Equation (13) expresses the fact that the modulation imposed at $z=0$ travels in each mode with the mode characteristic group velocity $V_{m}$; in particular, that frequency modulation, which affects the phase of $F(t)$, is ineffective in influencing the total power carried through a fiber section.

In order to satisfactorily treat the situation in which mode coupling is present, one has to resort to an ensemble-average approach. More precisely, one introduces a statistical ensemble of macroscopically equivalent fibers possessing microscopic random imperfections and then considers the ensemble average, indicated by the symbol $\langle\ldots\rangle$. Accordingly, all the significant quantities will be expressed by means of two statistically independent averaging operations, the first of which, indicated by \langle\rangle$_{\mathrm{ay}}$, refers to the source, while the second, indicated by angular brackets, is associated with the introduction of the statistical fiber ensemble. Thus one has, for example, the total average power carried through a fiber section given by

$$
\begin{aligned}
\left\langle P_{t}\right\rangle= & \sum_{m}\left\langle P_{m}(z, t)\right\rangle \\
= & P \sum_{m} \int d \omega \int d \omega^{\prime}\left\langle\left\langle c_{m}(z, \omega) c_{m}^{*}\left(z, \omega^{\prime}\right)\right\rangle_{\mathrm{av}}\right\rangle \\
& \times \exp \left\{i\left(\omega-\omega^{\prime}\right) t-i\left[\beta_{m}(\omega)-\beta_{m}\left(\omega^{\prime}\right)\right] z\right\} .
\end{aligned}
$$




\section{STATISTICAL THEORY OF TIME-DEPENDENT PROPAGATION IN AN ENSEMBLE OF FIBERS}

In order to study the space-time evolution of $\left\langle P_{m}(z, i)\right\rangle$ in the fiber, one has to, according to Eq. (14), investigate the behavior of the quantity $\left\langle\left\langle c_{m}(z, \omega) c_{m}^{*}\left(z, \omega^{\prime}\right)\right\rangle_{\text {av }}\right\rangle$. The starting point is the set of equations describing the deterministic evolution of the $c_{m}$ 's in a single fiber. They read ${ }^{1}$

$$
\frac{d c_{m}(z, \omega)}{d z}=\sum_{j} A_{m j}\left(z, \omega, \omega_{0}\right) c_{j}(z, \omega), \quad m=1,2, \ldots,
$$

where

$$
\begin{aligned}
A_{m j}\left(z, \omega, \omega_{0}\right)= & K_{m j}\left(z, \omega_{0}\right) \\
& \times \exp \left\{i\left[\beta_{m}(\omega)-\beta_{j}(\omega)\right] z\right\},
\end{aligned}
$$

the $K_{m j}$ 's being suitable coupling coefficients obeying the relation

$$
K_{m j}=-K_{j m}^{*} .
$$

An equation completely analogous to Eq. (15) holds obviously true for $\omega^{\prime}$ and reads

$\frac{d c_{m}\left(z, \omega^{\prime}\right)}{d z}=\sum_{j} A_{m j}\left(z, \omega^{\prime}, \omega_{0}\right) c_{j}\left(z, \omega^{\prime}\right), \quad m=1,2, \ldots$.

From Eqs. (15) and (18) one can immediately derive the following set of equations:

$$
\begin{aligned}
& \frac{d}{d z}\left[c_{m}^{*}\left(z, \omega^{\prime}\right) c_{j}(z, \omega)\right] \\
& =\sum_{k}\left\{A_{j k}\left(z, \omega, \omega_{0}\right) c_{m}^{*}\left(z, \omega^{\prime}\right) c_{k}(z, \omega)\right. \\
& \left.\quad+A_{m k}^{*}\left(z, \omega^{\prime}, \omega_{0}\right) c_{j}(z, \omega) c_{k}^{*}\left(z, \omega^{\prime}\right)\right\}, m, j=1,2, \ldots .
\end{aligned}
$$

At this point, one can follow a procedure analogous to that introduced in the monochromatic case $\left(\omega=\omega^{\prime}\right)$ (Ref. 4); that is, one formally integrates Eq. (19) and then substitutes in the resulting expression for $c_{m}^{*}\left(z, \omega^{\prime}\right) c_{j}(z$, $\omega)$ the formal solution for the quantities $c_{m}^{*}\left(z, \omega^{\prime}\right) c_{k}(z$, $\omega)$ and $c_{j}(z, \omega) c_{k}^{*}\left(z, \omega^{\prime}\right)$. After doing this, one can perform the double-averaging operation on the resulting equations.

If the coupling process is stationary to order. two in the $z$ direction, that is,

$$
\left\langle K_{m j}\left(z, \omega_{0}\right) K_{r s}\left(z^{\prime}, \omega_{0}\right)\right\rangle=F_{m j r s}\left(\left|z-z^{\prime}\right|\right),
$$

and if

$$
\left\langle K_{m j}\left(z, \omega_{0}\right)\right\rangle=0,
$$

by assuming that the mode-field amplitudes do not appreciably vary over distances comparable with the typical correlation length $D$ of the correlation function defined in Eq. (20), one obtains

$$
\begin{aligned}
\frac{d}{d z} X_{m r}\left(z, \omega, \omega^{\prime}\right)= & \sum_{j} \sum_{k} X_{k r}\left(z, \omega, \omega^{\prime}\right) \exp \left\{i\left[\beta_{m}(\omega)-\beta_{k}(\omega)\right] z\right\} \int_{0}^{\infty}\left\langle K_{m j}\left(z^{\prime}\right) K_{j k}(0)\right\rangle \\
& \times \exp \left\{-i\left[\beta_{j}(\omega)-\beta_{k}(\omega)\right] z^{\prime}\right\} d z^{\prime}+X_{j k}\left(z, \omega, \omega^{\prime}\right) \exp \left\{i\left[\beta_{m}(\omega)-\beta_{j}(\omega)+\beta_{k}\left(\omega^{\prime}\right)-\beta_{r}\left(\omega^{\prime}\right)\right] z\right\} \\
& \times \int_{0}^{\infty}\left\langle K_{m j}\left(z^{\prime}\right) K_{r k}^{*}(0)\right\rangle \exp \left\{-i\left[\beta_{k}\left(\omega^{\prime}\right)-\beta_{r}\left(\omega^{\prime}\right)\right] z^{\prime}\right\} d z^{\prime}+X_{k j}\left(z, \omega, \omega^{\prime}\right) \\
& \times \exp \left\{i\left[\beta_{j}\left(\omega^{\prime}\right)-\beta_{r}\left(\omega^{\prime}\right)+\beta_{m}(\omega)-\beta_{k}(\omega)\right] z\right\} \int_{0}^{\infty}\left\langle K_{r j}^{*}\left(z^{\prime}\right) K_{m k}(0)\right\rangle \exp \left\{-i\left[\beta_{m}(\omega)-\beta_{k}(\omega)\right] z^{\prime}\right\} d z^{\prime} \\
& +X_{m k}\left(z, \omega, \omega^{\prime}\right) \exp \left\{i\left[\beta_{k}\left(\omega^{\prime}\right)-\beta_{r}\left(\omega^{\prime}\right)\right] z\right\} \int_{0}^{\infty}\left\langle K_{r j}^{*}\left(z^{\prime}\right) K_{j k}^{*}(0)\right\rangle \exp \left\{-i\left[\beta_{k}\left(\omega^{\prime}\right)-\beta_{j}\left(\omega^{\prime}\right)\right] z^{\prime}\right\} d z^{\prime}
\end{aligned}
$$

where we have defined

$$
X_{m r}\left(z, \omega, \omega^{\prime}\right)=\left\langle\left\langle c_{m}(z, \omega) c_{r}^{*}\left(z, \omega^{\prime}\right)\right\rangle_{\mathrm{av}}\right\rangle ;
$$

and the dependence on $\omega_{0}$ of the coupling coefficients has been omitted.

It can now be observed that only nonoscillatory exponential terms in $z$ contribute appreciably to the righthand side of Eq. (22), since integrals over $z$ of rapidly oscillating terms can be neglected with respect to integrals of slowly varying functions (rotating-wave approximation). ${ }^{7}$ More precisely, in order to benefit from this approximation, one has to assume that the $c_{m}$ 's do not undergo relevant variations over distances of the kind $\left[\beta_{m}(\omega)-\beta_{k}(\omega)\right]^{-1}$ and $\left[\beta_{m}(\omega)-\beta_{f}(\omega)+\beta_{r}(\omega)-\beta_{s}(\omega)\right]^{-1}$, unless, respectively, $m=k$ and $m=j, r=s$ or $m=s$, $j=r$. Under the same hypothesis, it follows that the $c_{m}$ 's do not vary over the distance $\left[\beta_{m}(\omega)-\beta_{j}(\omega)+\beta_{r}\left(\omega^{\prime}\right)\right.$ $\left.-\beta_{s}\left(\omega^{\prime}\right)\right]^{-1}$, unless $m=j, r=s$ or $m=s, j=r$, since

$\beta_{m}(\omega)-\beta_{j}(\omega)+\beta_{r}\left(\omega^{\prime}\right)-\beta_{s}\left(\omega^{\prime}\right) \simeq \beta_{m}(\omega)-\beta_{j}(\omega)+\beta_{r}(\omega)-\beta_{s}(\omega)$, provided that the condition $\left|\omega-\omega^{\prime}\right| / \omega \ll 1$ is fulfilled [see Eq. (3)]. Equation (24) can be justified by observing that

$$
\begin{aligned}
\beta_{m}(\omega) & -\beta_{j}(\omega)+\beta_{r}\left(\omega^{\prime}\right)-\beta_{s}\left(\omega^{\prime}\right) \\
\simeq & \beta_{m}(\omega)-\beta_{j}(\omega)+\beta_{r}(\omega)-\beta_{s}(\omega)+\left(\frac{d \beta_{r}}{d \omega}-\frac{d \beta_{s}}{d \omega}\right)\left(\omega^{\prime}-\omega\right),
\end{aligned}
$$

and that

$$
\begin{aligned}
& \left|\frac{\left(d \beta_{r} / d \omega-d \beta_{s} / d \omega\right)\left(\omega^{\prime}-\omega\right)}{\omega}\right| \\
& \quad \ll \frac{\left|\beta_{m}(\omega)-\beta_{j}(\omega)+\beta_{r}(\omega)-\beta_{s}(\omega)\right|}{\omega},
\end{aligned}
$$

Eq. (26) being always valid if the differences between the inverses of the group velocities are of the same order of the differences between the inverses of the phase velocities.

By taking advantage of the rotating-wave approximation, Eq. (22) yields 


$$
\begin{aligned}
& \frac{d}{d z} X_{m r}\left(z, \omega, \omega^{\prime}\right)=X_{m r}\left(z, \omega, \omega^{\prime}\right) \\
& \quad \times\left(\sum_{j} \int_{0}^{\infty}\left\langle K_{m j}\left(z^{\prime}\right) K_{j m}(0)\right\rangle \exp \left\{-i\left[\beta_{j}(\omega)-\beta_{m}(\omega)\right] z^{\prime}\right\} d z^{\prime}\right. \\
& \quad+\sum_{j} \int_{0}^{\infty}\left\langle K_{r j}^{*}\left(z^{\prime}\right) K_{j r}^{*}(0)\right\rangle \exp \left\{-i\left[\beta_{r}\left(\omega^{\prime}\right)-\beta_{j}\left(\omega^{\prime}\right)\right] z^{\prime}\right\} d z^{\prime} \\
& \left.\quad+\int_{-\infty}^{+\infty}\left\langle K_{m m}\left(z^{\prime}\right) K_{r r}^{*}(0)\right\rangle d z^{\prime}\right), \quad m \neq r .
\end{aligned}
$$

It is not difficult to show (see Appendix) that the coefficient of $X_{m r}$ on the right-hand side of Eq. (27) has a nonpositive real part, so that Eq. (27) can be rewritten

$$
\frac{d}{d z} X_{m r}\left(z, \omega, \omega^{\prime}\right)=-g_{m r} X_{m r}\left(z, \omega, \omega^{\prime}\right), \quad m \neq r,
$$

with $\operatorname{Reg}_{m r} \geq 0$, whose solution is

$$
X_{m r}\left(z, \omega, \omega^{\prime}\right)=\left\langle c_{m}(0, \omega) c_{r}^{*}\left(0, \omega^{\prime}\right)\right\rangle_{\mathrm{av}} e^{-z_{m r^{2}}}, \quad m \neq r,
$$

where $c_{m}(0, \omega) \equiv c_{m}(z=0, \omega)$.

Equation (29) shows that the cross-correlation terms in the mode-field amplitudes practically vanish after a distance of the order $1 / \operatorname{Re} g_{m r}$; or that they remain identically zero if they possess this value at $z=0$ (excitation by a spatially incoherent source).

For the diagonal terms $m=r$, we obtain from Eq. (22),

$$
\begin{aligned}
\frac{d}{d z} X_{m m}\left(z, \omega, \omega^{\prime}\right)= & -\left(\sum_{j \neq m} h_{m j}\right) X_{m m}\left(z, \omega, \omega^{\prime}\right) \\
& +\sum_{j \neq m} h_{m j} \exp \left\{i\left[\beta_{m}(\omega)-\beta_{m}\left(\omega^{\prime}\right)\right] z\right. \\
& \left.+i\left[\beta_{j}\left(\omega^{\prime}\right)-\beta_{j}(\omega)\right] z\right\} X_{j j}\left(z, \omega, \omega^{\prime}\right),
\end{aligned}
$$

where

$$
\begin{aligned}
h_{m j}= & \int_{-\infty}^{+\infty}\left\langle K_{m j}\left(z^{\prime}\right) K_{m j}^{*}(0)\right\rangle \\
& \times \exp \left\{i\left[\beta_{j}\left(\omega_{0}\right)-\beta_{m}\left(\omega_{0}\right)\right] z^{\prime}\right\} d z^{\prime}
\end{aligned}
$$

is a real non-negative quantity (see Appendix). In deriving Eq. (30) we have taken advantage of the fact that the value of $h_{m j}$ is not substantially modified by the substitution $\omega \rightarrow \omega_{0}$ and $\omega^{\prime}-\omega_{0}$ performed in writing Eq. (31).

The set of Eqs. (30) describes in full generality the evolution of the autocorrelation functions of the modefield amplitudes and allows in principle the evaluation of $\left\langle P_{t}(z, t)\right\rangle$ by means of Eq. (14). It is, however, more convenient to deal directly with a set of equations connecting the average powers $\left\langle P_{m}(z, t)\right\rangle$ carried by each mode. By recalling the expression of $\left\langle P_{m}(z, t)\right\rangle$ given by Eq. (14), one obtains, with the help of Eq. (30),

$$
\begin{aligned}
& \frac{\partial}{\partial z}\left\langle P_{m}(z, t)\right\rangle=-\left(\sum_{j \neq m} h_{m j}\right)\left\langle P_{m}(z, t)\right\rangle \\
& \quad+\sum_{j \neq m} h_{m j}\left\langle P_{j}(z, t)\right\rangle+P \int d \omega \int d \omega^{\prime} i\left[\beta_{m}\left(\omega^{\prime}\right)-\beta_{m}(\omega)\right] \\
& \left.\quad \times \exp \left[i\left(\omega-\omega^{\prime}\right) t\right] \exp \left\{-i\left[\beta_{m}(\omega)-\beta_{m}\left(\omega^{\prime}\right)\right] z\right\} X_{m m}\right)\left(z, \omega, \omega^{\prime}\right) .
\end{aligned}
$$

The last term on the right-hand side of Eq. (32) can be rewritten, with the help of Eq. (11),

$$
-\frac{1}{V_{m}} \frac{\partial}{\partial t}\left\langle P_{m}(z, t)\right\rangle,
$$

so that finally one has

$$
\begin{aligned}
\frac{\partial}{\partial z} & \left\langle P_{m}(z, t)\right\rangle+\frac{1}{V_{m}} \frac{\partial}{\partial t}\left\langle P_{m}(z, t)\right\rangle \\
& =\sum_{j} h_{m j}\left[\left\langle P_{j}(z, t)\right\rangle-\left\langle P_{m}(z, t)\right\rangle\right],
\end{aligned}
$$

which constitutes the set of coupled equations describing the propagation of a pulse in a multimode lightguide. At this stage, distributed losses can be easily incorporated by adding the term $-2 \alpha_{m}\left\langle P_{m}(z, t)\right\rangle$ on the right-hand side of Eq. (34).

The set of Eqs. (34), which describes the time-dependent propagation of the average modal powers, is identical with the one worked out by previous authors. ${ }^{1-3}$ The present derivation has the advantage of clearly showing that the limits of validity of Eq. (34) rely on the small-bandwidth assumption expressed by Eq. (3), as well as on the hypothesis of slow variation of the field amplitudes required also in the stationary case. ${ }^{1,4}$

\section{PROPAGATION OF A FREQUENCY-MODULATED SIGNAL}

The form of the set of Eqs. (34) implies that the average power $\left\langle P_{m}(z, t)\right\rangle$ carried by each mode is not in- fluenced by the frequency modulation of the input signal; that is, that this kind of modulation cannot be transmitted by the fiber, as long as one collects all the light coming out from the whole fiber section. This circumstance is no longer true if one considers the power transmitted through a smaller area $\sigma$ of the section. In this case, the orthogonality of the modes is no longer effective in canceling the cross-correlation terms between different modes, and the expression of the power $\left\langle P_{t}^{\sigma}\right\rangle$ carried through this area contains also nondiagonal contributions. More precisely, one has, according to Eq. (5),

$$
\left\langle P_{t}^{\sigma}\right\rangle=\frac{1}{2} \operatorname{Re} \sum_{m} \sum_{r} T_{m r},
$$

with

$$
\begin{aligned}
T_{m r}(z, t)= & \iint_{\sigma} d x d y \mathrm{E}_{m}\left(\mathrm{r}, \omega_{0}\right) \times \mathrm{H}_{r}^{*}\left(\mathrm{r}, \omega_{0}\right) \cdot \mathrm{e}_{z} \\
& \times \int d \omega \int d \omega^{\prime} \exp \left\{i\left(\omega-\omega^{\prime}\right) t\right. \\
& \left.-i\left[\beta_{m}(\omega)-\beta_{r}\left(\omega^{\prime}\right)\right] z\right\} X_{m r}\left(z, \omega, \omega^{\prime}\right) .
\end{aligned}
$$

Recalling Eq. (29), one can rewrite the nondiagonal contributions as

$$
\begin{aligned}
T_{m r}(z, t)= & \exp \left(-g_{m r} z\right) \iint_{\sigma} d x d y \mathrm{E}_{m}\left(\mathrm{r}, \omega_{0}\right) \times \mathrm{H}_{r}^{*}\left(\mathrm{r}, \omega_{0}\right) \cdot \mathrm{e}_{z} \\
& \times \int d \omega \int d \omega^{\prime} \exp \left\{i\left(\omega-\omega^{\prime}\right) t-i\left[\beta_{m}(\omega)-\beta_{r}\left(\omega^{\prime}\right)\right] z\right\} \\
& \times\left\langle c_{m}(0, \omega) c_{r}^{*}\left(0, \omega^{\prime}\right)\right\rangle_{\mathrm{av}}, m \neq r
\end{aligned}
$$


which shows that the $T_{m r}$ 's $(m \neq r)$ evolve as in the absence of mode coupling, except for the fact that their envelope undergoes an exponential decay with the fiber length $z$. In particular, if the source is spatially incoherent, then $T_{m r}(z, t)=0(m \neq r)$, and $\left\langle P_{t}^{\sigma}\right\rangle$ is not influenced by the modulation of the signal. If, conversely, the source possesses some degree of spatial coherence, then the $T_{m r}$ 's $(m \neq r)$ are sensibly different from zero over a certain fiber length, and Eqs. (9)-(12) can be recalled for determining their evolution, thus getting

$$
T_{m r}(z, t) \propto e^{-z_{m} z}\left\langle F\left(t-\frac{z}{V_{m}}\right) F^{*}\left(t-\frac{z}{V_{r}}\right)\right\rangle_{\mathrm{av}}, \quad m \neq r .
$$

If one assumes the exciting source to be a frequencymodulated single-mode laser, then

$$
F(t)=\exp \left[i \phi(t)+i \gamma t^{2}\right],
$$

where $\phi(t)$ is a normally distributed random function responsible for the laser linewidth, $\gamma$ is a real constant proportional to the frequency-modulation rate, and

$$
\begin{aligned}
T_{m r}(z, t) \propto & \exp \left(-g_{m r} z\right) \exp \left(-\frac{\left|1 / V_{m}-1 / V_{r}\right| z}{t_{c}}\right) \\
& \times \exp \left[i \gamma z^{2}\left(\frac{1}{V_{m}^{2}}-\frac{1}{V_{r}^{2}}\right)+2 i \gamma t\left(\frac{z}{V_{r}}-\frac{z}{V_{m}}\right)\right], m \neq r,
\end{aligned}
$$

$t_{c}=1 / \delta \nu$ being the coherence time of the source. Equation (40) shows that $T_{m r}$ goes to zero over the shortest of the two distances $1 / g_{m r}$ and $t_{c}\left|1 / V_{m}-1 / V_{r}\right|$, the latter being the traveled length over which two modes $m$ and $r$ started from $z=0$ at the same instant acquire a time delay larger than $t_{c} \cdot{ }^{8}$ On the fiber length over which the $T_{m}$ 's $(m \neq r)$ are still different from zero, their time dependence, which is unaffected by mode coupling, determines the time behavior of $\left\langle P_{t}^{0}\right\rangle$, since the diagonal terms $T_{m m}$, being proportional to $\left\langle P_{m}\right\rangle$, do not depend on time.

\section{BEHAVIOR OF FLUCTUATIONS IN THE NONSTATIONARY CASE}

In order to deal with practical situations, in which a single fiber is usually employed, it is desirable to evaluate the fluctuations of the relevant quantities around their average values. In the monochromatic case, this has been done ${ }^{1,4}$ by calculating for a lossless fiber the asymptotic value (in $z$ ) of the normalized variance

$$
\left(\left\langle P_{m}^{2}\right\rangle-\left\langle P_{m}\right\rangle^{2}\right) /\left\langle P_{m}\right\rangle^{2} \text {. }
$$

For large $z$, the resulting value is 1 , which implies a $100 \%$ uncertainty on the actual value of the $m$ th mode power in a single fiber. This rather disappointing situation drastically changes in the case of a stationary signal possessing a finite bandwidth $\delta \omega$, where it has been shown that the asymptotic normalized variance tends to vanish. ${ }^{9}$ In this section, we study the evolution of the fourth-order field-amplitude correlation functions relative to different modes and different frequencies, which will allow us to generalize the result of Ref. 9 to nonstationary situations.

The starting point is the set of Eqs. (19), which immediately furnishes

$$
\begin{aligned}
& \frac{d}{d z}\left\langle c_{m}(z, \omega) c_{r}^{*}\left(z, \omega^{\prime}\right)\right\rangle_{\mathrm{av}}\left\langle c_{s}\left(z, \omega^{\prime \prime}\right) c_{n}^{*}\left(z, \omega^{\prime \prime \prime}\right)\right\rangle_{\mathrm{av}} \\
& \quad=\sum_{j}\left\{A_{m j}(z, \omega)\left\langle c_{j}(z, \omega) c_{r}^{*}\left(z, \omega^{\prime}\right)\right\rangle_{\mathrm{av}}\left\langle c_{s}\left(z, \omega^{\prime \prime}\right) c_{n}^{*}\left(z, \omega^{\prime \prime \prime}\right)\right\rangle_{\mathrm{av}}+A_{r j}^{*}\left(z, \omega^{\prime}\right)\left\langle c_{m}(z, \omega) c_{j}^{*}\left(z, \omega^{\prime}\right)\right\rangle_{\mathrm{av}}\left\langle c_{s}\left(z, \omega^{\prime \prime}\right) c_{n}^{*}\left(z, \omega^{\prime \prime \prime}\right)\right\rangle_{\mathrm{av}}\right. \\
& \left.\quad+A_{s j}\left(z, \omega^{\prime \prime}\right)\left\langle c_{m}(z, \omega) c_{r}^{*}\left(z, \omega^{\prime}\right)\right\rangle_{\mathrm{av}}\left\langle c_{j}\left(z, \omega^{\prime \prime}\right) c_{n}^{*}\left(z, \omega^{\prime \prime \prime}\right)\right\rangle_{\mathrm{av}}+A_{n j}^{*}\left(z, \omega^{\prime \prime}\right)\left\langle c_{m}(z, \omega) c_{r}^{*}\left(z, \omega^{\prime}\right)\right\rangle_{\mathrm{av}}\left\langle c_{s}\left(z, \omega^{\prime \prime}\right) c_{j}^{*}\left(z, \omega^{\prime \prime \prime}\right)\right\rangle_{\mathrm{av}}\right\}
\end{aligned}
$$

It is worthwhile to emphasize at this point that the source-average \langle\rangle$_{\mathrm{av}}$ in $\mathrm{Eq}$. (42) operates on mode-amplitude pairs successively. In fact, the basic quantity measured by the detector in a single fiber is the modal power $P_{m}(z, t)$ [see Eq. (8)], which contains the second-order average $\left\langle c_{m}(z, \omega) c_{m}^{*}\left(z, \omega^{\prime}\right)\right\rangle_{\text {av }}$, so that the evolution of ensemble averages of the kind $\left\langle P_{m}(z, t) P_{n}(z, t)\right\rangle$ involves the fourth-order products $\left\langle c_{m}(z, \omega) c_{m}^{*}\left(z, \omega^{\prime}\right)\right\rangle_{\mathrm{av}}\left\langle c_{n}\left(z, \omega^{\prime \prime}\right) c_{n}^{*}\left(z, \omega^{\prime \prime \prime}\right)\right\rangle_{\mathrm{av}}$. In turn, the evolution of $\left\langle c_{m}(z, \omega) c_{m}^{*}\left(z, \omega^{\prime}\right)\right\rangle_{\text {av }}$ is connected with nondiagonal second-order products of the type $\left\langle c_{j}(z, \omega) c_{r}^{*}\left(z, \omega^{\prime}\right)\right\rangle_{\mathrm{av}}$, which justifies the appearance of \langle\rangle$_{\mathrm{av}}\langle\rangle_{\mathrm{av}}$ in Eq. (42).

By taking $r=m, s=n$ and $\omega=\omega^{\prime}, \omega^{\prime \prime}=\omega^{\prime \prime \prime}$, it is not difficult to obtain from Eq. (42), by means of a direct extension of the procedure outlines in Sec. III, the following set of equations:

$$
\begin{aligned}
\frac{d}{d z} & \left\langle\tilde{P}_{m}(z, \omega) \tilde{P}_{n}\left(z, \omega^{\prime \prime}\right)\right\rangle=-\left(\sum_{j} h_{m j}\right)\left\langle\tilde{P}_{m}(z, \omega) \tilde{P}_{n}\left(z, \omega^{\prime \prime}\right)\right\rangle+\sum_{j} h_{m}\left\langle\tilde{P}_{j}(z, \omega) \tilde{P}_{n}\left(z, \omega^{\prime \prime}\right)\right\rangle \\
& +\left(\left\langle\left\langle c_{n}^{*}\left(z, \omega^{\prime \prime}\right) c_{m}\left(z, \omega^{\prime \prime}\right)\right\rangle_{\mathrm{av}}\left\langle c_{n}(z, \omega) c_{m}^{*}(z, \omega)\right\rangle_{\mathrm{av}}\right\rangle \exp \left\{i\left[\beta_{m}(\omega)-\beta_{m}\left(\omega^{\prime \prime}\right)+\beta_{n}\left(\omega^{\prime \prime}\right)-\beta_{n}(\omega)\right] z\right\}\left(1-\delta_{m n}\right) \int_{0}^{\infty}\left\langle K_{m n}\left(z^{\prime}\right) K_{m n}(0)\right\rangle\right. \\
& \left.\times \exp \left\{-i\left[\beta_{n}\left(\omega^{\prime \prime}\right)-\beta_{m}\left(\omega^{\prime \prime}\right)\right] z^{\prime}\right\} d z^{\prime}+\mathrm{c} . \mathrm{c}\right)+\left(\sum_{j \neq m}\left\langle\left\langle c_{j}(z, \omega) c_{m}^{*}(z, \omega)\right\rangle_{\mathrm{av}}\left\langle c_{m}\left(z, \omega^{\prime \prime}\right) c_{j}^{*}\left(z, \omega^{\prime \prime}\right)\right\rangle_{\mathrm{av}}\right\rangle\right. \\
& \left.\times \exp \left\{i\left[\beta_{m}(\omega)-\beta_{m}\left(\omega^{\prime \prime}\right)+\beta_{j}\left(\omega^{\prime \prime}\right)-\beta_{j}(\omega)\right] z\right\} \delta_{n m} \int_{0}^{\infty}\left\langle K_{m j}\left(z^{\prime}\right) K_{m j}^{*}(0)\right\rangle \exp \left\{-i\left[\beta_{j}\left(\omega^{\prime \prime}\right)-\beta_{m}\left(\omega^{\prime \prime}\right)\right] z^{\prime}\right\} d z^{\prime}+\mathrm{c.c}\right)+N_{\mathrm{ex}},
\end{aligned}
$$


where $N_{\text {ex }}$ represents same terms with the exchange $m \leftrightarrow n, \omega-\omega^{\prime \prime}$, having defined

$$
\tilde{P}_{m}(z, \omega)=\left\langle\left|c_{m}(z, \omega)\right|^{2}\right\rangle_{\mathrm{av}} \cdot
$$

While the set of Eqs. (43) possesses a general validity under the usual hypotheses justyfying the rotating-wave approximation, it can be cast in a particularly simple form if the relation

$$
\left|\left(\frac{d \beta_{m}}{d \omega}-\frac{d \beta_{j}}{d \omega}\right)\left(\omega-\omega^{\prime \prime}\right)\right| z \gg 2 \pi, \quad j \neq m
$$

is fulfilled and if the field amplitudes do not appreciably vary over a distance $z_{c}^{(m, j)}$ given by [compare with Eq. $(40)]$

$$
z_{c}^{(m, j)}=\left|\left(1 / V_{m}-1 / V_{j}\right)\right|^{-1} 2 \pi / \delta \omega .
$$

In fact, in this case, one can again take advantage of the rotating-wave approximation on the right-hand side of Eq. (43), which reduces to

$$
\begin{aligned}
\frac{d}{d z}\left\langle\tilde{P}_{m}(z, \omega) \tilde{P}_{n}\left(z, \omega^{\prime \prime}\right)\right\rangle \\
=\sum_{j} h_{m j}\left[\left\langle\tilde{P}_{j}(z, \omega) \tilde{P}_{n}\left(z, \omega^{\prime \prime}\right)\right\rangle-\left\langle\tilde{P}_{m}(z, \omega) \tilde{P}_{n}\left(z, \omega^{\prime \prime}\right)\right\rangle\right] \\
\quad+\sum_{j} h_{n j}\left[\left\langle\tilde{P}_{j}\left(z, \omega^{\prime \prime}\right) \tilde{P}_{m}(z, \omega)\right\rangle-\left\langle\tilde{P}_{n}\left(z, \omega^{\prime \prime}\right) \tilde{P}_{m}(z, \omega)\right\rangle\right] .
\end{aligned}
$$

From Eq. (47), in the case $m=n$ and for values of $z$ large enough so its left-hand side vanishes, it is immediate to deduce the following asymptotic equality:

$$
\begin{aligned}
& \left\langle\tilde{P}_{j}(z, \omega) \tilde{P}_{m}\left(z, \omega^{\prime \prime}\right)\right\rangle_{a}+\left\langle\tilde{P}_{j}\left(z, \omega^{\prime \prime}\right) \tilde{P}_{m}(z, \omega)\right\rangle_{a} \\
& \quad=2\left\langle\tilde{P}_{m}(z, \omega) \tilde{P}_{m}\left(z, \omega^{\prime \prime}\right)\right\rangle_{a},
\end{aligned}
$$

where the subscript $a$ stands for asymptotic. On the other hand, energy conservation along the fiber requires that

$$
\sum_{j} \tilde{P}_{j}(z, \omega)=\sum_{j} \tilde{P}_{j}(0, \omega) \text { for every } \omega,
$$

which in turn implies

$$
\begin{gathered}
\sum_{m}\left\langle\tilde{P}_{m}(z, \omega) \tilde{P}_{m}\left(z, \omega^{\prime \prime}\right)\right\rangle+\frac{1}{2} \sum_{m \neq j} \sum_{j}\left\langle\tilde{P}_{j}(z, \omega) \tilde{P}_{m}\left(z, \omega^{\prime \prime}\right)\right\rangle \\
+\frac{1}{2} \sum_{m \neq j} \sum_{j}\left\langle\tilde{P}_{j}\left(z, \omega^{\prime \prime}\right) \tilde{P}_{m}(z, \omega)\right\rangle \\
=\sum_{m} \tilde{P}_{m}(0, \omega) \sum_{j} \tilde{P}_{j}\left(0, \omega^{\prime \prime}\right),
\end{gathered}
$$

a relation which asymptotically yields, with the help of Eq. (48),

$\sum_{m}\left\langle\tilde{P}_{m}(z, \omega) \tilde{P}_{m}\left(z, \omega^{\prime \prime}\right)\right\rangle_{a}+N(N-1)\left\langle\tilde{P}_{m}(z, \omega) \tilde{P}_{m}\left(z, \omega^{\prime \prime}\right)\right\rangle_{a}$

$$
=\sum_{m} \tilde{P}_{m}(0, \omega) \sum_{j} \tilde{P}_{j}\left(0, \omega^{\prime \prime}\right),
$$

where $N$ is the total number of guided modes. Recalling now the asymptotic equipartition condition, expressing the fact that, in a lossless case, the power per mode is the same in every mode for $z$ sufficiently large, ${ }^{1}$

$$
\left\langle\tilde{P}_{m}(z, \omega)\right\rangle_{a}=\frac{1}{N} \sum_{j} \tilde{P}_{j}(0, \omega) \text { for every } \omega,
$$

Eq. (51) can be rewritten

$$
\begin{aligned}
\sum_{m} & \left\langle\tilde{P}_{m}(z, \omega) \tilde{P}_{m}\left(z, \omega^{\prime \prime}\right)\right\rangle_{a}+N(N-1)\left\langle\tilde{P}_{m}(z, \omega) \tilde{P}_{m}\left(z, \omega^{\prime \prime}\right)\right\rangle_{a} \\
& =N^{2}\left\langle\tilde{P}_{m}(z, \omega)\right\rangle_{a}\left\langle\tilde{P}_{m}\left(z, \omega^{\prime \prime}\right)\right\rangle_{a},
\end{aligned}
$$

from which follows the relevant expression

$$
\left\langle\tilde{P}_{m}(z, \omega) \tilde{P}_{m}\left(z, \omega^{\prime \prime}\right)\right\rangle_{a}=\left\langle\tilde{P}_{m}(z, \omega)\right\rangle_{a}\left\langle\tilde{P}_{m}\left(z, \omega^{\prime \prime}\right)\right\rangle_{a} \text {. }
$$

We now observe that the energy $I_{m}(z)$ pertaining to the $m$ th mode

$$
I_{m}(z)=\int_{-\infty}^{+\infty} P_{m}(z, t) d t
$$

can be expressed by means of Eqs. (8) and (44) as

$$
I_{m}(z)=2 \pi P \int \tilde{P}_{m}(z, \omega) d \omega
$$

The normalized variance of the statistical quantity $I_{m}$ can be accordingly written

$$
\begin{aligned}
& \frac{\left\langle I_{m}^{2}\right\rangle-\left\langle I_{m}\right\rangle^{2}}{\left\langle I_{m}\right\rangle^{2}}=\left(\int d \omega \int d \omega^{\prime \prime}\left\langle\tilde{P}_{m}(z, \omega) \tilde{P}_{m}\left(z, \omega^{\prime \prime}\right)\right\rangle\right. \\
& \left.\quad-\int d \omega \int d \omega^{\prime \prime}\left\langle\tilde{P}_{m}(z, \omega)\right\rangle\left\langle\tilde{P}_{m}\left(z, \omega^{\prime \prime}\right)\right\rangle\right) \\
& \quad \times\left(\int d \omega \int d \omega^{\prime \prime}\left\langle\tilde{P}_{m}(z, \omega)\right\rangle\left\langle\tilde{P}_{m}\left(z, \omega^{\prime \prime}\right)\right\rangle\right)^{-1} .
\end{aligned}
$$

Since, for $z \gg z_{c}^{(m, j)}$, most couples of frequencies in the relevant integration domain fulfill Eq. (45), Eq. (54) applies and, consequently, the normalized variance tends asymptotically to vanish, that is

$$
\left(\left\langle I_{m}^{2}\right\rangle_{a}-\left\langle I_{m}\right\rangle_{a}^{2}\right) /\left\langle I_{m}\right\rangle_{a}^{2}=0 \text {. }
$$

This implies that no statistical uncertainty is present in $I_{m}$, so that equipartition of the total energy among the various modes takes place in the single fiber. As a particular case of this general statement, the asymptotic power $P_{m}$ has the same property in the stationary regime, as it has been shown elsewhere, ${ }^{9}$ since in this situation $I_{m}$ and $P_{m}$ are proportional.

The complete investigation of the behavior of fluctuations in the nonstationary regime requires the analysis of the quantities $\left\langle P_{m}^{2}(z, t)\right\rangle$ and $\left\langle P_{m}(z, t) P_{n}(z, t)\right\rangle$. To this end, the system of Eqs. (42) has to be specified to the case $r=m, s=n$, since

$$
\begin{aligned}
\left\langle P_{m}(z, t) P_{n}(z, t)\right\rangle= & P^{2} \int d \omega \int d \omega^{\prime} \int d \omega^{\prime \prime} \int d \omega^{\prime \prime \prime} \exp \left[i\left(\omega-\omega^{\prime}\right) t\right] \exp \left\{i\left(\omega^{\prime \prime}-\omega^{\prime \prime \prime}\right) t-i\left[\beta_{m}(\omega)-\beta_{m}\left(\omega^{\prime \prime}\right)\right] z\right. \\
& \left.-i\left[\beta_{n}\left(\omega^{\prime \prime}\right)-\beta_{n}\left(\omega^{\prime \prime \prime}\right)\right] z\right\}\left\langle\left\langle c_{m}(z, \omega) c_{m}^{*}\left(z, \omega^{\prime}\right)\right\rangle_{\mathrm{av}}\left\langle c_{n}\left(z, \omega^{\prime \prime}\right) c_{n}^{*}\left(z, \omega^{\prime \prime \prime}\right)\right\rangle_{\mathrm{av}}\right\rangle .
\end{aligned}
$$

By means of the general procedure followed in this paper, it is possible to deduce a set of equations governing the evolution of $\left\langle\left\langle c_{m}(z, \omega) c_{m}^{*}\left(z, \omega^{\prime}\right)\right\rangle_{\text {av }}\left\langle c_{n}\left(z, \omega^{\prime \prime}\right) c_{n}^{*}\left(z, \omega^{\prime \prime \prime}\right)\right\rangle_{\mathrm{av}}\right\rangle$. In order to simplify the notation, let us define 
$Y_{m r}\left(z, \omega, \omega^{\prime}\right)=\exp \left[i \beta_{r}\left(\omega^{\prime}\right) z-i \beta_{m}(\omega) z\right]\left\langle c_{m}(z, \omega) c_{r}^{*}\left(z, \omega^{\prime}\right)\right\rangle_{\mathrm{av}}$

The final form of the closed system of equations then reads

$\frac{d}{d z}\left\langle Y_{m m}\left(z, \omega, \omega^{\prime}\right) Y_{n n}\left(z, \omega^{\prime \prime}, \omega^{\prime \prime \prime}\right)\right\rangle=i\left[\beta_{m}\left(\omega^{\prime}\right)-\beta_{m}(\omega)+\beta_{n}\left(\omega^{\prime \prime \prime}\right)-\beta_{n}\left(\omega^{\prime \prime}\right)\right]\left\langle Y_{m m}\left(z, \omega, \omega^{\prime}\right) Y_{n n}\left(z, \omega^{\prime \prime}, \omega^{\prime \prime \prime}\right)\right\rangle$

$$
\begin{aligned}
& -\left[\sum_{k}\left(h_{k m}+h_{k n}\right)\right]\left\langle Y_{m m}\left(z, \omega, \omega^{\prime}\right) Y_{n n}\left(z, \omega^{\prime \prime}, \omega^{\prime \prime \prime}\right)\right\rangle+\sum_{k} h_{k n}\left\langle Y_{m m}\left(z, \omega, \omega^{\prime}\right) Y_{k k}\left(z, \omega^{\prime \prime}, \omega^{\prime \prime \prime}\right)\right\rangle \\
& +\sum_{k} h_{k m}\left\langle Y_{k k}\left(z, \omega, \omega^{\prime}\right) Y_{n n}\left(z, \omega^{\prime \prime}, \omega^{\prime \prime \prime}\right)\right\rangle-\left(1-\delta_{n m}\right) h_{m n}\left[\left\langle Y_{n m}\left(z, \omega, \omega^{\prime}\right) Y_{m n}\left(z, \omega^{\prime \prime}, \omega^{\prime \prime \prime}\right)\right\rangle\right. \\
& \left.+\left\langle Y_{m n}\left(z, \omega, \omega^{\prime}\right) Y_{n m}\left(z, \omega^{\prime \prime}, \omega^{\prime \prime \prime}\right)\right\rangle\right]+\delta_{n m}\left(\sum_{k \neq m} h_{m k}\left[\left\langle Y_{k m}\left(z, \omega, \omega^{\prime}\right) Y_{m k}\left(z, \omega^{\prime \prime}, \omega^{\prime \prime \prime}\right)\right\rangle+\left\langle Y_{m k}\left(z, \omega, \omega^{\prime}\right) Y_{k m}\left(z, \omega^{\prime \prime}, \omega^{\prime \prime \prime}\right)\right\rangle\right]\right)
\end{aligned}
$$

and

$$
\begin{aligned}
\frac{d}{d z} & \left\langle Y_{m r}\left(z, \omega, \omega^{\prime}\right) Y_{r m}\left(z, \omega^{\prime \prime}, \omega^{\prime \prime \prime}\right)\right\rangle=i\left[\beta_{r}\left(\omega^{\prime}\right)-\beta_{m}(\omega)+\beta_{m}\left(\omega^{\prime \prime \prime}\right)-\beta_{r}\left(\omega^{\prime \prime}\right)\right]\left\langle Y_{m r}\left(z, \omega, \omega^{\prime}\right) Y_{r m}\left(z, \omega^{\prime \prime}, \omega^{\prime \prime \prime}\right)\right\rangle \\
& -\left(\sum_{k \neq r} h_{k r}+\sum_{k \neq m} h_{k m}\right)\left\langle Y_{m r}\left(z, \omega, \omega^{\prime}\right) Y_{r m}\left(z, \omega^{\prime \prime}, \omega^{\prime \prime \prime}\right)\right\rangle+\sum_{k \neq r} h_{r k}\left\langle Y_{m k}\left(z, \omega, \omega^{\prime}\right) Y_{k m}\left(z, \omega^{\prime \prime}, \omega^{\prime \prime \prime}\right)\right\rangle+\sum_{k \neq m} h_{m k}\left\langle Y_{k r}\left(z, \omega^{\prime} \omega^{\prime}\right)\right. \\
& \left.\times Y_{r k}\left(z, \omega^{\prime \prime}, \omega^{\prime \prime \prime}\right)\right\rangle-h_{m r}\left[\left\langle Y_{r r}\left(z, \omega, \omega^{\prime}\right) Y_{m m}\left(z, \omega^{\prime \prime}, \omega^{\prime \prime \prime}\right)\right\rangle+\left\langle Y_{m m}\left(z, \omega, \omega^{\prime}\right) Y_{r r}\left(z, \omega^{\prime \prime}, \omega^{\prime \prime \prime}\right)\right\rangle\right], m \neq r .
\end{aligned}
$$

The set of Eqs. (61) and (62) represents, together with the set of Eqs. (30), a fairly complete analytical description of nonstationary propagation. In fact, while Eqs. (30) concern the evolution of the average power per mode, Eqs. (61) and (62) allow one to describe the fluctuations around this quantity in terms of fourth-order averages. One can observe that, in the general case, it is not possible to obtain a system of equations containing only the quantities $\left\langle P_{m} P_{n}\right\rangle$, while this is possible in the monochromatic stationary propagation. ${ }^{4}$ This is related to the essential role of the average over the source, which does not allow the nondiagonal terms $\left\langle Y_{m r}\left(\omega, \omega^{\prime}\right)\right.$ $\left.\times Y_{r m}\left(\omega^{\prime \prime}, \omega^{\prime \prime \prime}\right)\right\rangle$ to be rearranged as products $\left\langle Y_{m m}(\omega\right.$, $\left.\left.\omega^{\prime \prime \prime}\right) Y_{r r}\left(\omega^{\prime \prime}, \omega^{\prime}\right)\right\rangle$.

\section{CONCLUSIONS}

We have derived a set of statistical coupled equations describing the time-dependent propagation of a finitebandwidth carrier, which can be either amplitude or frequency modulated, placing into evidence the limits of validity of the approach, which involve both the value of the bandwidth and the variation of the mode-amplitude coefficients. In particular, the time evolution of a frequency-modulated signal does not depend on mode coupling, thus possessing a behavior completely different from that of an amplitude-modulated signal.

We have set in a rigorous form a theorem describing the smoothing of mode-power fluctuations over the fiber ensemble, due to the finite bandwidth, so as to include mode-energy fluctuations in nonstationary situations.

The general case of time-dependent propagation of fluctuations has been also considered, and a closed system of equations enabling to describe it has been derived.

\section{APPENDIX}

If one defines

$$
F_{m j}=\left(\frac{1}{L}\right)^{1 / 2} \int_{-L / 2}^{L / 2} d z K_{m j}(z) \exp \left[i\left(\beta_{j}-\beta_{m}\right) z\right],
$$

one can write

$$
\begin{aligned}
\left|F_{m j}\right|^{2}= & \frac{1}{L} \int_{-L / 2}^{L / 2} d z^{\prime} \int_{-L / 2}^{L / 2} d z\left\langle K_{m j}(z) K_{m j}^{*}\left(z^{\prime}\right)\right\rangle \\
& \times \exp \left[i\left(\beta_{j}-\beta_{m}\right)\left(z-z^{\prime}\right)\right],
\end{aligned}
$$

so that, remembering Eq. (31), and taking advantage of the finite range of the correlation function

$$
\left\langle K_{m j}(z) K_{m j}^{*}\left(z^{\prime}\right)\right\rangle=\left\langle K_{m j}\left(z-z^{\prime}\right) K_{m j}^{*}(0)\right\rangle,
$$

one has

$$
h_{m j}=\lim _{L \rightarrow \infty}\left|F_{m j}\right|^{2} \geq 0 \text {. }
$$

Let us now consider Eqs. (27) and (28). Since the quantity $\left\langle K_{m j}(z) K_{j m}(0)\right\rangle$ is a real even function of $z$, one can write

$$
\begin{aligned}
\operatorname{Reg}_{m r}= & \frac{1}{2} \sum_{j \neq m} h_{m j}+\frac{1}{2} \sum_{j \neq r} h_{j r} \\
& +\frac{1}{2} \lim _{L \rightarrow \infty}\left\langle\left|\left(\frac{1}{L}\right)^{1 / 2} \int_{-L / 2}^{L / 2} d z\left[K_{m m}(z)-K_{r r}(z)\right]\right|^{2}\right\rangle \geq 0 .
\end{aligned}
$$

*Research supported by the U. S. Army Research Office under Contract No. DAAG29-77-G-0130.

'On leave of absence from Fondazione Ugo Bordoni, Roma, Italy.

${ }^{1} \mathrm{D}$. Marcuse, Theory of Dielectric Optical Waveguides (Academic, New York, 1974).

${ }^{2}$ S. D. Personick, "Two Derivations of the Time-Dependent Coupled-Power Equations," Bell Syst. Tech. J. 54, 47-52 (1975).

${ }^{3}$ R. Steinberg, "Pulse Propagation in Multimode Fibers with Frequency-Dependent Coupling," IEEE Trans. Microwave Theory Tech. MTT-23, 121-122 (1975).

${ }^{4}$ B. Crosignani, B. Daino, and P. Di Porto, "Statistical Coupled Equations in Lossless Optical Fibers," IEEE Trans. Microwave Theory Tech. MTT-23, 416-420 (1975).

${ }^{5}$ See, for example, C. H. Papas, Theory of Electromagnetic Wave Propagation (McGraw-Hill, New York, 1965). 
${ }^{6}$ See, for example, A. Blanc-Lapierre and R. Fortet, Théorie des Fonctions Aléatoires (Masson et Cie; Paris, 1953).

${ }^{7}$ See, for example, M. Sargent III, M. O. Scully, and W. E. Lamb, Jr., Laser Physics (Addison-Wesley, Reading, Mass. 1974).

${ }^{8}$ B. Crosignani, B. Daino, and P. Di Porto, "Interference of
Mode Patterns in Optical Fibers, " Opt. Commun. 11, 178179 (1974).

${ }^{9}$ B. Crosignani, B. Daino, and P. Di Porto, "Smoothing of the Mode-Power Fluctuations in Lossless Optical Fibers Due to the Source Spectral Bandiwidth," Opt. Commun. 18, 551-552 (1976).

\title{
Longitudinal and transverse displacements of a bounded microwave beam at total internal reflection*
}

\author{
J. J. Cowan ${ }^{\dagger}$ \\ Oak Ridge National Laboratory, Oak Ridge, Tennessee 37830 \\ and Ion Physics Laboratory, Boris Kidrič Institute, Belgrade 11000, Yugoslavia \\ B. Aničin ${ }^{\ddagger}$ \\ Ion Physics Laboratory, Boris Kidrič Institute, Belgrade 11000, Yugoslavia \\ (Received 10 July 1976; revision received 25 July 1977)
}

\begin{abstract}
Longitudinal and transverse shifts of an $8 \mathrm{~cm}$ parallel bounded beam of $34.2 \mathrm{GHz}(8.77 \mathrm{~mm})$ microwaves totally reflected from a paraffin prism have been investigated. The $45^{\circ}-45^{\circ}-90^{\circ}$ prism is $18 \mathrm{~cm}$ high by $25 \mathrm{~cm}$ on the sides and the index of refraction is 1.491 . Longitudinal shifts as large as $3 \mathrm{~cm}$ have been measured in a single reflection near the critical angle for a beam linearly polarized in the plane of incidence. The shift for perpendicular polarization is approximately half this value. The results are in general agreement with the classical theory for the Goos-Hänchen effect. An incident beam polarized at $45^{\circ}$ to the incidence plane produces both parallel and perpendicular polarization shifts with values similar to the above. The shifts for both polarizations are reduced but are still distinctly separate if either a second prism or a metallic reflector is brought into the evanescent wave at millimeter distances from the interface. These results are in accordance with stationary phase calculations for two interfaces. It is found that a small $(6 \mathrm{~mm})$ transverse shift results if the prism is illuminated with circularly polarized microwaves.
\end{abstract}

\section{INTRODUCTION}

A bounded beam of light that enters a prism and is totally reflected at the prism-vacuum. interface is known to be displaced longitudinally from the position of geometrical reflection. The displacement is small, usually on the order of the illuminating wavelength, and it is larger for light polarized within the plane of incidence than perpendicular to it. This phenomenon, commonly known as the Goos-Hänchen shift, ${ }^{1}$ has been the object of considerable theoretical and experimental studies over the past few years, ${ }^{2}$ not only because of its importance in research on the fundamental properties of light but also because of its relation and application to the expanding fields of optical imaging ${ }^{3}$ and integrated optics. ${ }^{4}$

Theoretical treatments of the effect have stressed the important role played by the inhomogeneous, evanescent wave associated with total reflection that appears on the vacuum side of the interface. Accurate determinations of the shift have been made on the assumption that energy from the beam flows into the evanescent wave, travels along the surface for a certain distance and then passes back into the reflected beam. ${ }^{5}$ Other treatments, such as the stationary phase theory, implicitly take the surface wave into account through Fresnel's equations. ${ }^{6}$ Still other theories explain the shift in terms of a time delay of scattering process. ${ }^{7}$

In the usual beam-shift experiments light is linearly polarized either parallel or perpendicular to the plane of incidence, but there have been some interesting variations using circularly polarized and unpolarized light. With circular polarization the beam is shifted transversely, out of the plane of incidence. The direction depends on the sense of polarization, and the magnitude is smaller than that for the longitudinal shift. ${ }^{8}$ The transverse shift has been given as evidence for the noncollinarity of velocity and momentum of a spinning photon. ${ }^{9}$

Using unpolarized light two distinct longitudinal shifts occur, the larger corresponding to parallel polarization, and the smaller, to perpendicular polarization, with no intermediate values. ${ }^{10}$ Some authors have been able to explain this experiment on the basis of existing electromagnetic theory, ${ }^{11}$ while others have cited it as evidence that the photon has a small but finite mass. ${ }^{12}$

There are many interesting questions to be investigated and resolved with total reflection phenomena, but the experiments using visible light have not always been easy to perform. Since the shift is so small, special experimental techniques to amplify it have generally been required. This involves allowing the beam to be reflected many times before emerging from the prism, and then delicate measurement techniques are employed.

Experiments carried out at microwave frequencies, using wavelengths of about $1 \mathrm{~cm}$, are more amenable to analysis and interpretation and probably easier than those done in the optical region where the wavelength is much 